\title{
Genomic selection strategies for clonally propagated
}

\section{2 crops}

4 Gregor Gorjanc*, John M. Hickey*

5

*The Roslin Institute and Royal (Dick) School of Veterinary Studies, University of Edinburgh, Easter Bush Research Centre, Midlothian EH25 9RG, UK

Key message: For genomic selection in clonal breeding programs to be effective, crossing parents should be selected based on genomic predicted cross performance unless dominance is negligible. Genomic prediction of cross performance enables a balanced exploitation of the additive and dominance value simultaneously. A two-part breeding program with parent selection based on genomic predicted cross performance to rapidly drive population improvement has great potential to improve breeding clonally propagated crops. 


\section{Abstract}

For genomic selection in clonal breeding programs to be effective, crossing parents should be selected based on genomic predicted cross performance unless dominance is negligible. Genomic prediction of cross performance enables a balanced exploitation of the additive and dominance value simultaneously. Here, we compared different strategies for the implementation of genomic selection in clonal plant breeding programs. We used stochastic simulations to evaluate six combinations of three breeding programs and two parent selection methods. The three breeding programs included i) a breeding program that introduced genomic selection in the first clonal testing stage, and ii) two variations of a two-part breeding program with one and three crossing cycles per year, respectively. The two parent selection methods were i) selection of parents based on genomic estimated breeding values, and ii) selection of parents based on genomic predicted cross performance. Selection of parents based on genomic predicted cross performance produced faster genetic gain than selection of parents based on genomic estimated breeding values because it substantially reduced inbreeding when the dominance degree increased. The two-part breeding programs with one and three crossing cycles per year using genomic prediction of cross performance always produced the most genetic gain unless dominance was negligible. We conclude that i) in clonal breeding programs with genomic selection, parents should

40 be selected based on genomic predicted cross performance, and ii) a two-part breeding 41 program with parent selection based on genomic predicted cross performance to rapidly 42 drive population improvement has great potential to improve breeding clonally 43 propagated crops. 
In this paper we show that, for genomic selection in clonal breeding programs to be effective, crossing parents should be selected based on genomic predicted cross performance, unless dominance is negligible. In most plant and animal breeding programs which apply genomic selection, new parents are selected based on their genomic estimated breeding value (e.g. Meuwissen et al., 2016; Crossa et al., 2017). The genomic estimated breeding value (commonly referred to as GEBV) is by

51 definition the sum of the average effects predicted for all marker alleles of a genotype, while dominance deviation, which cannot be directly passed on to the progeny, is not considered (Goddard, 2009; Su et al., 2012). Selection based on the genomic estimated breeding value aids breeders in increasing the frequency of alleles with beneficial additive genetic effects in a given breeding population. As a result, heterozygosity is reduced. Although selection for the genomic estimated breeding value will increase the additive value over time, it may lead to a reduction of the dominance value, unless dominance is negligible. In the long term, using the genomic estimated breeding value to select new parents in breeding programs which deliver outbred varieties, such as in clonal plant breeding programs, might not be the optimal method to use in order to maximize the total genetic value of the breeding population in a sustainable fashion. roots and tubers, are clonally propagated (Grüneberg et al., 2009; Bradshaw, 2016). In clonal breeding programs, new genotypes are created by sexual reproduction and multiplied through clonal propagation (Bisognin, 2011; Gemenet and Khan, 2017). The new genotypes are first tested as seedlings in unreplicated trials during the initial phase 67 of the breeding program. Clonal propagation creates genetically identical plants from 
selected seedlings, which enables the testing of genotypes in clonal plots, using multiple replications, environments and years.

in their breeding population. As the testing progresses, the number of genotypes is successively reduced and those remaining are tested more intensively at increasingly higher numbers. The selected genotypes are used to achieve two specific objectives:

i) Generation of an improved offspring population via recombination of selected parents.

ii) Release of the best performing genotypes as improved clonal varieties.

several years. Traditionally, selection is based on phenotypic performance and the next generation's parents are selected in the later testing stages of the breeding program, which results in a long generation interval (Bradshaw, 2016), even in species with short generational times, such as strawberry.

Genomic selection offers great potential to optimize the process of identification of the best clones for varietal development, as well as the selection of new crossing parents. Genomic selection exploits associations between genomic markers and phenotypes to predict the value of genotypes based on their genomic marker profiles (Goddard and Hayes, 2007). The implementation of genomic selection provides three key advantages:

i) The generation interval can be reduced, since new parents can be selected as soon as they are genotyped.

ii) The selection accuracy can be increased, especially in early testing stages 
of a breeding program where the number of replications and environments is low.

iii) The selection intensity can be increased, for example by genotyping and predicting more genotypes than could be tested in the field. breeding programs. For example, in the context of breeding programs to develop inbred lines, Gaynor et al. (2017) presented a two-part breeding program employing genomic selection, which reorganized a plant breeding program into:

i) A population improvement component to develop improved germplasm through rapid recurrent genomic selection, and genotypes for varietal development. genetic gain relative to a conventional breeding program without increasing cost. genomic selection would allow breeders to minimize the generation interval and could substantially increase selection accuracy at the seedling stage.

109 can be selected as soon as the seedlings are genotyped. For example, the generation

110 interval in conventional strawberry breeding programs can be four to five years due to

111 the time it takes for testing to generate sufficient phenotypic records to accurately assess

112 a genotype. Genomic selection applied in the seedling stage could result in up to five

113 times the genetic gain achieved in a conventional strawberry breeding program in the 
114 same amount of time if the impact of the three other factors in the breeder's equation

115 (i.e., selection intensity, diversity and selection accuracy) remained constant.

The selection accuracy in the seedling stage could be increased since genomic

117 selection allows seedlings to be selected based on their predicted performance as clones

118 instead of their phenotypic performance per se. This is achieved when the genomic

119 selection model to select seedlings is trained using clonal phenotypes. In clonal

120 breeding programs, the seedling stage represents a severe genetic bottleneck; in

121 conventional strawberry breeding programs only a few hundred genotypes among

$12210,000-20,000$ unreplicated seedlings are selected and tested as clones. Selection

123 accuracy is extremely low at the seedling test stage for three reasons (Grüneberg et al.,

124 2009), which are:

i) Seedlings and clones with the same genotype can differ in their morphology and performance.

ii) Seedlings and clones are often grown in different environments. For example, in European strawberry breeding programs, seedlings are grown in matted rows on the soil and clones are grown as single pot plants on highly controlled table top systems.

iii) Single plant assessment of mostly general appearance and/or a few key traits in the seedling stage shows low heritability and has low correlation with the breeding goal trait (e.g., yield).

Replacing phenotypic selection in the seedling stage with genomic selection based on the predicted performance as clones eliminates all three challenges in one step. It also allows for early evaluation of important traits that are typically not evaluated until later testing stages of the breeding program, e.g. flavour and shelf life. 
139 of breeding programs which implement genomic selection. The genotypes in clonally

140 propagated crops are typically heterozygous. The genetic value of heterozygous

141 genotypes is a function of additive and non-additive gene action (Falconer and Mackay,

142 1996). If, for the sake of simplicity, epistasis is ignored, the non-additive gene action is

143 entirely defined by dominance. Whilst the differences in the genetic values between

144 genotypes are based on both additive and non-additive genetic effects, the additive

145 genetic variation is the crucial component which defines long-term genetic gain in a

146 breeding population subjected to recurrent selection (Bradshaw 2016). Hence, breeders

147 face the challenging task of having to increase the additive value over time while

148 simultaneously maintaining the dominance value via selection and recombination of

149 the best parents. The relative importance of these two targets is a function of the

150 dominance degree at the loci affecting the trait under consideration, which is mostly

151 unknown.

We hypothesise that genomic prediction of cross performance is a better method

153 to select new parents in a clonal breeding program than using the genomic estimated

154 breeding value. When genomic prediction of cross performance is used, pairs of parents

155 are selected based on the expectation of the total genetic value of their progeny.

156 Genomic prediction of cross performance could allow breeders to simultaneously

157 increase the frequency of alleles with beneficial additive effects and maintain

158 heterozygosity in the population to exploit dominance effects. In the long term, using

159 genomic prediction of cross performance to select new parents in a clonal breeding

160 program could be an effective method to sustainably maximize the total genetic value

161 of the breeding population. 
163 programs and two parent selection methods to deploy genomic selection in breeding

164 clonally propagated crops under different dominance degrees. The three breeding

165 programs included:

166

168 genomic predicted cross performance produced faster genetic gain than parent selection

i) A breeding program that introduced genomic selection in the first clonal testing stage, and

ii) Two variations of a two-part breeding program (Gaynor et al., 2017) with one and three crossing cycles per year, respectively.

The two parent parental selection methods were:

i) Selection of parents based on genomic estimated breeding values, and

ii) Selection of parents based on genomic predicted cross performance.

The six combinations of breeding program and parent selection method were compared to a conventional breeding program using phenotypic selection.

We observed that the breeding programs using selection of parents based on based on genomic estimated breeding values unless dominance was negligible. The highest rates of genetic gain were generated by the two-part breeding programs with parent selection based on genomic predicted cross performance. 


\section{Materials and methods}

185 programs and two parent selection methods to deploy genomic selection in breeding

186 clonally propagated crops with diploid (-like) meiotic behaviour. Therefore, we

187 simulated a quantitative trait representing yield under four different dominance degrees

188 and evaluated the long-term efficacy of the six combinations of breeding programs and

189 parent selection methods compared to a conventional breeding program using

190 phenotypic selection.

i) Genome simulation: a heterozygous genome sequence was simulated for a hypothetical diploid and clonally propagated crop species.

ii) Simulation of founder genotypes: the simulated genome sequences were used to generate a base population of 60 diploid founder genotypes. 
The simulation of the breeding programs comprised:

i) Recent (burn-in) breeding phase: a conventional phenotypic selection breeding program for clonally propagated crops was simulated for a period of 20 years (burn-in) to provide a common starting point for the future breeding phase.

ii) Future breeding phase: six combinations of three breeding programs and two parent selection methods to deploy genomic selection in clonally propagated crops were simulated and compared to the conventional breeding program for 20 years of breeding. In detail, we describe:

a. The genomic selection model used for genomic prediction.

b. The two parent selection methods including parent selection based on genomic estimated breeding values and parent selection based on genomic predicted cross performance.

c. The three breeding programs with genomic selection including a breeding program which implemented genomic selection in the clonal testing stage 1 , and two variations of a two-part breeding program which implemented genomic selection in the seedling stage with one and three crossing cycles per year, respectively. value in clonal testing stage 1. 


\section{Simulation of the founder genotype population}

\section{Genome simulation}

A heterozygous genome sequence was simulated for each genotype of a

232 hypothetical diploid and clonally propagated crop species. The simulated genome consisted of 20 chromosome pairs with a physical length of 108 base pairs and a genetic

234 length of 100 centiMorgans (cM), resulting in a total genetic length of 2,000 cM 235 comparable to that of the Fragaria $\times$ ananassa genome (Sargent et al., 2009, 2016; van 236 Dijk et al., 2014; Bassil et al., 2015). The chromosome sequences were generated using 237 the Markovian coalescent simulator (MaCS; Chen et al. 2009), which was deployed 238 using AlphaSimR version 0.11.0 (Gaynor et al., 2019). Recombination rate was derived 239 as ratio between genetic length and physical genome length (i.e., $100 \mathrm{cM} / 108$ base 240 pairs $=10-8$ ). The per-site mutation rate was set to $2.5 \times 10-8$ mutations per base pair. 241 Effective population size $\left(\mathrm{Ne}_{\mathrm{e}}\right)$ was set to 100 and resulted from a simulated coalescence 242 process with an effective population size of 500, 1,250, 1,500, 3,500, 6,000, 12,000 and 243100,000 set for 100, 500, 1,000, 5,000, 10,000, and 100,000 generations ago.

244 Successive reduction of the effective population size was used to reflect a progressive 245 restriction of genetic variation due natural and artificial selection. 
252 (QTN) and 1,000 single nucleotide polymorphisms (SNP) were randomly sampled

253 along each chromosome to simulate a quantitative trait that was controlled by 20,000

254 QTN and a SNP marker array with 20,000 markers.

Genetic values for a single trait representing yield were simulated by summing

258 the genetic effects at the 20,000 randomly sampled QTN. Three types of biological

259 effects were modelled at each QTN to simulate genetic values: additive effects,

260 dominance effects and genotype-by-environment effects. Under the AlphaSimR

261 framework, this is referred to as an ADG trait. We will give only a brief summary of

262 the modelling procedure, while a detailed description can be found in the vignette of

263 the AlphaSimR package (Gaynor et al., 2019).

Additive effects $(a)$ were sampled from a standard normal distribution and

265 scaled to obtain an additive variance of $\sigma_{A}^{2}=1$ in the founder population. Genotype-

266 by-environment effects were modelled using an environmental covariate and a

267 genotype-specific slope. The environmental covariate represented the environmental

268 component of the genotype-by-environment interaction and was sampled for each year

269 of the simulation from a standard normal distribution. The genotype-specific slope

270 represented the genetic component of the genotype-by-environment interaction. The

271 effects for the genotype specific slope were sampled from a standard normal

272 distribution and scaled to obtain a genotype-by-environment interaction variance of

$273 \sigma_{G x Y}^{2}=2 \sigma_{A}^{2}=2$ in the founder population. 
275 value of its additive effect $a_{i}$ by a locus-specific dominance degree $\delta_{i}$. A dominance

276 degree of 0 represents no dominance and a dominance degree of 1 represents complete

277 dominance. Dominance degrees between 0 and 1 correspond to partial dominance, and

278 values above 1 correspond to over-dominance. Dominance degrees were sampled from

279 a normal distribution with mean dominance coefficient $\mu_{\delta}$ and variance $\sigma_{\delta}^{2}$ :

$$
\delta_{i} \sim N\left(\mu_{\delta}, \sigma_{\delta}^{2}\right)
$$

284 simulate positive directional dominance and compared to zero dominance (i.e., additive 285 genetic control). The variance $\sigma_{\delta}^{2}$ was set to 0.2 . The dominance variance $\left(\sigma_{D}^{2}\right)$ was then 286 calculated based on the simulated dominance effects.

\section{Simulation of phenotypes}

Phenotypes for yield were generated by adding random error to the genetic

290 value of a genotype. The random error was sampled from a normal distribution with 291 mean zero and an error variance $\sigma_{e}^{2}$ defined by the target level of heritability at each testing stage of the breeding program. In the founder population, entry-mean values for 
narrow-sense heritabilities in later testing stages increased as a result of an increased

were calculated using the following equation:

$$
h^{2}=\frac{\sigma_{A}^{2}}{\sigma_{P}^{2}}=\frac{\sigma_{A}^{2}}{\sigma_{A}^{2}+\sigma_{D}^{2}+\sigma_{e}^{2} / n}
$$

\section{Simulation of the breeding programs} phenotypic selection was simulated for a period of 20 years (burn-in) to provide a common starting point for the future breeding phase. Each year of the conventional breeding program started with a crossing block of 60 parental genotypes. These genotypes were crossed to generate new seedlings, followed by a six year evaluation

307 period that involved six stages of testing. Selection of new parents and selection of the 308 best clones in each testing stage were based on phenotypic records. The structure and 309 the values for key parameters of the conventional breeding program were guided by a 310 commercial strawberry breeding program in the United Kingdom. Table 1 presents the 311 number of tested genotypes and replications for each testing stage of the conventional 312 breeding program as shown in Figure. 1.

314 in phase, six cycles of crossing and selection were conducted prior to the burn-in phase.

315 Each of these six cycles started with the same 60 founder genotypes to generate $150 \mathrm{~F}_{1}$ - 
316 families with 100 seedlings each, using random sampling of bi-parental crosses without

317 replacement. Starting from the set of 15,000 seedlings after the first crossing cycle, the

318 best genotypes were advanced one stage per cycle using phenotypic selection until each

319 testing stage was filled with a set of genotypes. Replacement of parents was omitted

320 during the filling of the breeding pipeline. This was done to ensure that total genetic

321 variance in the founder genotypes remained unchanged until the actual burn-in phase 322 started.

Table 1 Number of tested genotypes, replications and heritabilities used in the

324 conventional breeding program

\begin{tabular}{llccc}
\hline Year & Stage & Tested genotypes & Reps & $\begin{array}{c}\text { Narrow-sense } \\
\text { heritability }\left(h_{2}\right)^{*}\end{array}$ \\
\hline $\mathbf{1}$ & Seedlings & 15,000 & 1 & 0.10 \\
$\mathbf{2}$ & Clonal stage 1 & 1,000 & 1 & 0.30 \\
$\mathbf{3}$ & Clonal stage 2 & 100 & 2 & 0.46 \\
$\mathbf{4}$ & Clonal stage 3 & 20 & 4 & 0.63 \\
$\mathbf{5}$ & Clonal stage 4 & 5 & 6 & 0.72 \\
$\mathbf{6}$ & Clonal stage 5 & 5 & 6 & 0.72 \\
\hline
\end{tabular}

*entry-mean values based on the $\sigma_{A}^{2}: \sigma_{P}^{2}$ ratio in the founder population

In the burn-in phase, selection of new parents was carried out in the clonal

328 testing stages 2, 3, 4 and 5. Each year, the 30 genotypes in the crossing block with the 329 poorest per se performance were replaced by new parents. At first, all 30 genotypes in 330 the clonal testing stages 3,4 and 5 were added to the crossing block as new parents if 
331 they were not already represented. The remaining free slots in the crossing block were

332 filled with the best genotypes from the clonal testing stage 2.

The future breeding phase was used to evaluate six combinations of two

336 breeding programs and two parent selection methods to deploy genomic selection in

337 clonally propagated crops under different dominance degrees. These six combinations

338 were simulated for an additional 20 years of breeding and compared to the conventional

339 breeding program. The two genomic selection breeding programs included a

340 conventional breeding program with genomic selection which introduced genomic

341 selection in clonal testing stage 1 (Fig. 1), and two variations of a two-part breeding

342 program which introduced genomic selection in the seedling stage with one and three

343 crossing cycles per year, respectively (Fig. 2). The two parent selection methods were

344 selection of new parents based on genomic estimated breeding values, and selection of

345 new parents based on genomic predicted of cross performance. In order to obtain

346 approximately equal annual operating costs, the number of seedlings was reduced in

347 the two breeding programs with genomic selection to compensate for the additional

348 costs of genotyping. Estimated costs were set to $\$ 20$ for phenotypic evaluation and $\$ 25$

349 for array genotyping per genotype after consultation with strawberry breeders. Table 2

350 shows the number of crosses and seedlings per year for the conventional breeding

351 program and the three breeding programs with genomic selection. 
Table 2 Number of crosses per year and seedlings per cross, total number of seedlings and annual costs of the simulated breeding programs (Conv, conventional

356 breeding program; Conv GS, conventional breeding program with genomic selection;

357 2Part, two-part breeding program)

\begin{tabular}{lcccc}
\hline Breeding Program & $\begin{array}{c}\text { Crosses / } \\
\text { year }\end{array}$ & $\begin{array}{c}\text { Seedlings / } \\
\text { cross }\end{array}$ & $\begin{array}{c}\text { Seedlings } \\
\text { (total) }\end{array}$ & Costs (\$) \\
\hline Conv & 150 & 100 & 15,000 & 300,000 \\
Conv GS & 150 & 91 & 13,650 & 298,000 \\
2Part & 130 & 84 & 11,960 & 299,000 \\
2Part with 3 cycles & $100 \times 3$ & $40 \times 3$ & 12,000 & 300,000 \\
\hline
\end{tabular}

Genomic predictions were calculated using a ridge regression model (RR361 BLUP) including year as a fixed effect, additive and dominance SNP effects, and a 362 covariate accounting for directional dominance (or inbreeding depression) based on 363 average individual heterozygosity as described in detail by Xiang et al. (2016). The 364 effect estimated for the covariate accounting for directional dominance was divided by 365 the number of SNPs and added to the SNP-specific dominance effects. To obtain 366 genomic estimated breeding values, the predicted additive and dominance SNP effects 367 at each marker locus were used to calculate the average effect of an allele substitution 368 for each SNP (Varona et al., 2018), and all the substitution effects were summed. To 369 obtain genomic estimated genetic values, the predicted additive and dominance SNP 370 effects at each marker locus were summed. The initial training population at the start 
371 of the future breeding phase consisted of all the genotypes from clonal testing stage 1

372 of the last three years of the burn-in phase. The training population included 3,000

373 genotypes and 3,220 phenotypic records. In every year of the future breeding phase,

3741,000 new genotypes from clonal testing stage 1 were added to the training population.

Two parent selection methods were compared for the selection and crossing of new parents in the two breeding programs with genomic selection. The first parent selection method will be referred to as parent selection based on genomic estimated

380 breeding values. This method represented a conventional "good by good" crossing 381 scheme. The genotypes with the highest genomic estimated breeding values were 382 selected as new parents and used to completely replace the previous year's crossing 383 block. Crossing was implemented as random sampling of bi-parental combinations 384 without replacement. The second parent selection method will be referred to as parent 385 selection based on genomic predicted cross performance. This method implemented 386 systematic selection of bi-parental crosses. The best bi-parental crosses were selected 387 based on the predicted mean genetic values of the $\mathrm{F}_{1}$ of a cross. In this way, the average 388 amount of heterosis predicted for the $\mathrm{F}_{1}$ due to complementarity between two parents 389 was directly considered in the parent selection process. The mean genetic value of the $390 \quad$ F1 of a cross was predicted using the following equation given by Falconer \& Mackay 391 (1996):

$$
M_{F_{1}}=a(p-q-y)+d[2 p q+y(p-q)]
$$


394 additive and dominance effects of the SNP markers, $p$ and $q$ being the marker allele

395 frequencies of one parent and $y$ representing the difference of gene frequency between

396 the two parents. The concept of the crossing block was abandoned.

The conventional breeding program with genomic selection introduced

401 program with genomic selection is shown in Figure 1. All 1,000 genotypes in clonal

402 testing stage 1 were genotyped and phenotyped to serve as the training population for

403 the genomic selection model. When parents were selected based on genomic estimated

404 breeding values, each year the best 60 genotypes in clonal testing stage 1 were used to

405 replace the whole crossing block. When parents were selected based on genomic

406 predicted cross performance, bi-parental cross performance was predicted for all

407 pairwise combinations between the genotypes in clonal testing stage 1 . The generation

408 interval was two years. Genomic selection was also used to advance the best 100 clones

409 from clonal testing stage 1 to clonal testing stage 2 based on their genomic estimated

410 genetic value. 
415 through rapid recurrent genomic selection, and a product development component to

416 identify the best performing genotypes. Two variations of the two-part breeding

417 program with one and three crossing cycles per year respectively were simulated. The

418 structure of the two-part breeding programs is shown in Figure 2. Genomic selection

419 was introduced in the seedling stage. All seedlings were genotyped and phenotypic

420 selection in the seedling stage was entirely replaced by genomic selection. All 1,000

421 genotypes in clonal testing stage 1 were genotyped and phenotyped to serve as the

422 training population for the genomic selection model. Thus, a key feature of the two-

423 part breeding program is that seedlings were selected using a prediction model that was

424 trained with phenotypic records from clones. When parents were selected based on

425 genomic estimated breeding values, in each crossing cycle the best 60 seedlings were

426 used to replace the whole crossing block. When parents were selected based on genomic

427 predicted cross performance, bi-parental cross performance was predicted for all

428 pairwise combinations between the seedlings. The generation interval was one year

429 with one crossing cycle per year and 1/3 year with 3 crossing cycles per year. Genomic

430 selection was also used to advance the best 1,000 seedlings to clonal testing stage 1 and

431 the best 100 clones from clonal testing stage 1 to clonal testing stage 2 based on their

432 genomic estimated genetic value.

The performance of the three breeding programs and the two parent selection

436 methods in comparison to the conventional breeding program was evaluated by

437 measuring the mean total genetic value in clonal testing stage 1. Each evaluation 
438 included ten simulation runs. The mean total genetic value was measured in clonal 439 testing stage 1 for two reasons:

i) It was the earliest testing stage in which clones were evaluated.

ii) The general trends observed for genetic gain in clonal testing stage 1 were over time were also measured in clonal testing stage 1 . The genomic inbreeding coefficient was calculated as the percentage increase of homozygosity at all quantitative trait nucleotides relative to the average homozygosity observed in the founder population.

All breeding programs were compared for total genetic variance, additive variance and dominance variance over time, results are shown in the supplementary material (Fig. S1-S3).

i) Prediction accuracy was assessed in the three breeding programs with genomic selection as the accuracy of the parent selection method including parent selection based on genomic estimated breeding values and parent selection based on genomic predicted cross performance.

ii) Prediction accuracy was assessed as the prediction accuracy of the total genetic value in the seedling stage, which was used to advance seedlings to clonal testing stage 1 . 


\section{Results}

The results show that for genomic selection in a clonal breeding program to be

463 effective, crossing parents should be selected based on genomic predicted cross

464 performance unless dominance is negligible. Selection of parents based on genomic

465 predicted cross performance produced faster genetic gain than selection of parents

466 based on genomic estimated breeding values when the dominance degree was greater

467 than zero (Fig. 3). As the dominance degree increased, selection of parents using 468 genomic prediction of cross performance also produced increasingly more genetic gain 469 than selection based on genomic estimated breeding values. The two variations of the 470 two-part breeding program using genomic prediction of cross performance always 471 produced the most genetic gain unless dominance was negligible. However, while the 472 two-part breeding program with three crossing cycles per year produced the most genetic gain when the dominance degree was low, the two-part breeding program with one crossing cycle per year produced the most genetic gain when the dominance degree

475 was high. The breeding programs using selection of parents based on genomic 476 estimated breeding values on the other hand, produced negative genetic gain when the 477 dominance degree was high. Selection of parents based on genomic prediction of cross 478 performance was advantageous over selection of parents based on genomic estimated 479 breeding values because it substantially reduced inbreeding in the breeding population 480 when the dominance degree increased (Fig. 4). This enabled a better exploitation of the 481 additive value and the dominance value simultaneously, which became more important 482 as the dominance degree increased (Fig. 5). Additionally, selection of parents based on 483 genomic prediction of cross performance became more accurate and selection of 
parents based on genomic estimated breeding values became less accurate at higher dominance degrees (Fig. 6).

\section{Genetic gain}

Selection of parents based on genomic predicted cross performance produced faster genetic gain than selection of parents based on genomic estimated breeding values unless dominance was negligible. This is shown in Figure 3, which plots genetic gain as the mean genetic value against time in clonal testing stage 1 . The four panels show genetic gain under the different simulated dominance degrees for four types of breeding programs and two types of parent selection. As the dominance degree increased, selection of parents based on genomic prediction of cross performance produced increasingly more genetic gain than selection based on genomic estimated breeding values.

The three genomic selection breeding programs using genomic prediction of cross performance always produced more genetic gain than the conventional breeding program. The two variations of the two-part breeding program using genomic prediction of cross performance always produced the most genetic gain unless dominance was negligible (Fig. 3). However, while the two-part breeding program with three crossing cycles per year produced the most genetic gain when the dominance degree was 0.1 and 0.3 , the two-part breeding program with one crossing cycle per year produced the most genetic gain when the dominance degree was 0.9. When the dominance degree was 0.1 , the two-part breeding program gave 2.8 times the genetic gain of the conventional breeding program with one crossing cycle per year, and more 
507 than three times the genetic gain with three crossing cycles per year. When the

508 dominance degree was 0.9 , it gave almost 7 times the genetic gain of the conventional

509 breeding program with one crossing cycle per year, and more than five times the genetic

510 gain with three crossing cycles per year.

Figure 3 also shows that the two-part breeding program with parent selection

512 based on genomic estimated breeding values and three crossing cycles per year

513 generated the most genetic gain when the dominance degree was zero. However, after

514 a sharp increase in the first few years, the rate of genetic gain drastically decreased and

515 started to approach a plateau. The two-part breeding program with parent selection

516 based on genomic estimated breeding values and one crossing cycle per year generated

517 the second most genetic gain. In the first few years it showed a lower rate of genetic

518 gain than both variations of the two-part breeding program using genomic prediction

519 of cross performance. In the long term, however, both two-part breeding programs

520 using genomic prediction of cross performance started to plateau and were

521 outperformed by the two-part breeding program with parent selection based on genomic

522 estimated breeding values and one crossing cycle per year.

Figure 3 also shows that selection of parents based on genomic estimated

524 breeding values produced negative genetic gain over time when the dominance degree

525 was high. All breeding programs showed a reduced rate of genetic gain when the

526 dominance degree increased. However, this reduction was stronger when new parents

527 were selected based on genomic estimated breeding values. Both variations of the two-

528 part breeding program with parent selection based on genomic estimated breeding

529 values produced even less genetic gain than the conventional breeding program when

530 the dominance degree was 0.3 and 0.9 . These results were not surprising as selection of 
531 parents based on genomic estimated breeding values gave a faster increase in the

532 inbreeding coefficient than selection of parents based on genomic predicted cross

533 performance when the dominance degree was high, which resulted in inbreeding

534 depression.

\section{Genomic inbreeding coefficient}

Selection of parents based on genomic predicted cross performance

538 substantially reduced inbreeding when the dominance degree increased. This is shown

539 in Figure 4, which plots the genomic inbreeding coefficient against time in clonal

540 testing stage 1 . The four panels show the inbreeding coefficient under the different

541 simulated dominance degrees. As the dominance degree increased, all breeding

542 programs showed a decreased growth rate of the genomic inbreeding coefficient.

543 However, this decrease was much stronger when parents were selected based on

544 genomic predicted cross performance compared to when genomic estimated breeding

545 values were used.

Figure 4 also shows that the two-part breeding programs with selection of

547 parents based on genomic predicted cross performance gave the strongest reduction in

548 the genomic inbreeding coefficient as the dominance degree increased. When the

549 dominance degree was zero, both breeding programs had almost approached complete

550 inbreeding at the end of the future breeding phase. However, when the dominance

551 degree was 0.9 , the two-part breeding program with parent selection based on genomic

552 predicted cross performance and one crossing cycle per year gave the lowest inbreeding

553 coefficient, which was negative during the entire future breeding phase. The two-part 
554 breeding program with parent selection based on genomic predicted cross performance

555 and three crossing cycles per year was also negative in the first half of the future

556 breeding phase, but showed a slightly faster increase and became positive during the

557 second half. By reducing the growth rate of the inbreeding coefficient when the

558 dominance degree increased, selection of cross performance directly took the

559 increasing importance of dominance effects to the total genetic value into account.

\section{Additive values and dominance values}

Selection of parents based on genomic predicted cross performance enabled a better exploitation of the combined additive and dominance values than did selection

564 of parents based on genomic estimated breeding values. This is shown in Figure 5, 565 which plots the additive values and the dominance values against time in clonal testing 566 stage 1. The three upper panels (a-c) show the additive values and the three lower panels 567 (d-f) show the dominance values.

The two-part breeding program with parent selection based on genomic 569 predicted cross performance and three crossing cycles per year gave the highest 570 increase of the additive value over time when the dominance degree was 0.1 and 0.3 571 (Fig. 5a-c). The two-part breeding program with parent selection based on genomic 572 estimated breeding values and three crossing cycles per year gave a lower additive 573 value, as growth rate showed a stronger reduction over time and approached a plateau 574 towards the end of the future breeding phase. However, when the dominance degree 575 was 0.9 , it gave the highest increase of the additive value. 
Figure 5 a-c also shows that the rate of increase of the additive value over time

577 was reduced as the dominance degree increased. All breeding programs gave a lower

578 additive value under high dominance degrees compared to when the dominance degree

579 was low. The conventional breeding program always gave the lowest increase of the

580 additive value.

Selection of parents using genomic prediction of cross performance generated

582 increased dominance values as the dominance degree increased (Fig. 5d-f). It gave a

583 reduction of the dominance value when the dominance degree was 0.1 , but a strong

584 initial increase when the dominance degree was 0.9. The increase of the dominance

585 value compensated for the reduced rate of increase of the additive value as the

586 dominance degree increased. The two-part breeding program with parent selection

587 based on genomic predicted cross performance and one crossing cycle per year gave

588 the strongest increase. When the dominance degree was high, the two-part breeding

589 program with one crossing cycle per year and the conventional breeding program with

590 genomic selection maintained a relatively stable, positive dominance value over the

591 entire future breeding phase. The two-part breeding program with three crossing cycles

592 per year, however, showed a continuous reduction of the dominance value over time. It

593 also showed a faster reduction than the other two breeding programs when the

594 dominance degree was 0.1 and 0.3 .

Selection of parents based on genomic estimated breeding values did not

596 effectively exploit the dominance value as the dominance degree increased. This is also

597 shown in Figure 5 d-f. Both variations of the two-part breeding program with parent

598 selection based on genomic estimated breeding values generated reduced dominance

599 values as the dominance degree increased. This reduction in the dominance value over 
600 time became more extreme as the dominance degree increased, and was greater than

601 the increase in the additive value over time when the dominance degree was high.

607 substantially higher prediction accuracy when the dominance degree was high. At

608 higher dominance degrees, selection of parents based on genomic predicted cross

609 performance became more accurate and selection of parents based on genomic

610 estimated breeding values became less accurate. This is shown in Figure 6, which plots

611 the prediction accuracy of the parent selection methods against time. The two panels

612 show prediction accuracy under the dominance degrees of 0.1 and 0.9 for the three

613 types of genomic selection breeding programs and two types of parent selection

614 method. Prediction accuracy of the parent selection method was measured in the

615 seedling stage for the two-part breeding programs and in clonal testing stage 1 for the

616 conventional breeding program with genomic selection. Prediction accuracy of

617 genomic predicted cross performance became more similar in the three genomic

618 selection breeding programs when the dominance degree increased. 
623 value in the seedling stage over time. The two panels show prediction accuracy under

624 the dominance degrees of 0.1 and 0.9 . The two-part breeding program with parent 625 selection based on genomic estimated breeding values and one crossing cycle per year 626 always showed the highest prediction accuracy. Prediction accuracy was lower when 627 parents were selected based on genomic predicted cross performance compared to 628 genomic estimated breeding values. It also was lower when three crossing cycles per 629 year were used compared to one crossing cycle. The difference in prediction accuracy 630 due to the number of crossing cycles per year, however, became smaller as the 631 dominance degree increased. The conventional breeding program with genomic 632 selection using genomic predicted cross performance to select parents showed the 633 lowest prediction accuracies under all dominance degrees.

\section{Discussion}

For genomic selection in clonal breeding programs to be effective, crossing

637 parents should be selected based on genomic predicted cross performance unless 638 dominance is negligible. To discuss this result, we first describe how genomic selection 639 can improve clonal breeding programs under the assumption of additive genetic control.

640 We show that the two-part breeding program enables effective exploitation of genomic 641 selection in breeding clonally propagated crops. We also explain that under additive 642 genetic control, differences in genetic gain between the two parent selection methods 
644 on genomic predicted cross performance compared to selection of parents based on

645 genomic estimated breeding values. After the discussion of results when traits were

646 under additive genetic control, we explain why genomic selection of new parents

647 requires consideration of dominance effects unless dominance is negligible. We show

648 that selection of parents based on genomic predicted cross performance enables

649 efficient simultaneous exploitation of additive and dominance effects, which facilitates

650 exploitation of pseudo-overdominance in the progeny of a cross when the dominance

651 degree is high. We also show that multiple crossing cycles per year can have an adverse

652 effect on long-term genetic gain, especially when the dominance degree is high. We

653 then explain that, at higher dominance degrees, heterozygosity becomes a reliable

654 predictor of the dominance value when parents are selected based on genomic predicted

655 cross performance. Finally, we conclude that genomic prediction of cross performance

656 could be an efficient method to select parents not only in clonal plant breeding

657 programs, but also in other breeding programs for outbred individuals including animal

658 breeding programs.

659

661 genetic control

662

Under additive genetic control, genomic selection of new parents always

663 produced faster genetic gain than phenotypic selection of new parents. This was

664 observed regardless of whether parents were selected based on genomic estimated

665 breeding values or based on genomic predicted cross performance. 
667 of genetic variance into genetic gain in both variations of the two-part breeding program

668 with one and three crossing cycles per year, respectively, and in the conventional

669 breeding program with genomic selection. This improvement resulted from a shortened

670 generation interval and an increased selection accuracy in early selection stages. As a

671 consequence, the breeding programs with genomic selection also showed an

672 accelerated depletion of genetic variance over time compared to the conventional

673 breeding program (Fig. S1). This depletion was most severe when three crossing cycles

674 per year were used, and it caused genetic gain to approach a plateau in the second half 675 of the future breeding phase.

Our findings under additive genetic control were consistent with those of

677 Gaynor et al. (2017) who used stochastic simulations to evaluate genomic selection

678 strategies in plant breeding programs for developing inbred lines. We refer the reader

679 to this study for a detailed description of the relationship between the generation

680 interval, prediction accuracy and genetic variance when additive genetic control is 681 assumed. conventional breeding program with genomic selection under additive genetic control 
689 based genomic predicted cross performance and three crossing cycles per year, and

690 three times the genetic gain of the conventional breeding program when used with

691 parent selection based genomic estimated breeding values and three crossing cycles per

692 year. The increased rates of genetic gain compared to the conventional breeding

693 program resulted from a very short generation interval and an improved selection

694 accuracy in the seedling stage.

Selection in the seedling stage poses a major challenge in clonal breeding

696 programs due to a high selection intensity combined with low selection accuracy

697 (Grüneberg et al., 2009; Bradshaw, 2016). The two-part breeding programs improved

698 selection accuracy by replacing phenotypic selection with genomic selection, which

699 enabled improvements in the selection criterion for seedlings. When phenotypic

700 selection was used, seedlings were selected based on their observed per se performance.

701 When genomic selection was used, seedlings were selected based on their predicted

702 performance as clones because the genomic selection model was trained using data

703 from the clonal testing stages.

i) The phenotypic records in the clonal stages which were used to train the selection model had a higher heritability than the phenotypic records of the unreplicated seedlings.

ii) Marker alleles were replicated within and across years. 
711 of new parents in the seedling stage, allowing for one or multiple cycles of crossing per

712 year to minimize the length of the breeding cycle.

The conventional breeding program with genomic selection gave 1.7 times the

714 genetic gain of the conventional breeding program when parents were selected based

715 on genomic estimated breeding values and 1.9 times the genetic gain when parents were

716 selected based on genomic predicted cross performance. Genomic selection was

717 applied in clonal testing stage 1 and selection in the seedling stage was based on

718 phenotypic per se performance. Hence, selection accuracy in the seedling stage was not

719 improved compared to the conventional breeding program. The increased rate of 720 genetic gain mainly resulted from a shortened generation interval and an improved 721 selection accuracy in clonal testing stage 1. increased selection intensity compared to selection of parents based on genomic parent selection methods mainly resulted from an increased selection intensity when parents were selected based on genomic predicted cross performance compared to selection of parents based on genomic estimated breeding values. 
733 predicted mean genetic values of the $\mathrm{F}_{1}$ of a bi-parental cross. Under additive genetic

734 control, the predicted mean genetic value of the $\mathrm{F}_{1}$ is equal to the mean genomic

735 estimated breeding value of both parents. Selection of parents based on genomic

736 predicted cross performance resulted in the excessive use of a few very good parents in

737 many crosses. As a consequence, the selection intensity for parents was higher

738 compared to when parents were selected based on genomic estimated breeding values

739 and randomly crossed.

In the conventional breeding program with genomic selection, this increase in

741 selection intensity resulted in more genetic gain over time compared to when parents

742 were selected based on genomic estimated breeding values. In the two-part breeding

743 programs, it resulted in more genetic gain in the first years, but thereafter genetic gain

744 reached a plateau due to a depletion of genetic variance. This depletion of genetic

745 variance was more severe when three crossing cycles per year were used.

A crossing strategy in a practical breeding program would probably lie

747 somewhere in between the two simulated parent selection methods. A breeder would

748 not randomly select crosses, but rather combine parents that are expected to generate

749 improved progeny. Although very good genotypes may be used at high frequency, a

750 breeder would make sure that an overly excessive use did not restrict the genetic

751 variation in long-term. 
756 additive and non-additive gene action. If epistasis is ignored, the non-additive gene

757 action is entirely determined by dominance. Achieving a high rate of genetic gain then

758 depends on an efficiently balanced exploitation of the additive and dominance effects

759 (Bradshaw, 2016).

In particular, this requires two opposed actions:

i) The frequency of alleles with beneficial additive genetic effects in homozygous state has to be increased to improve the additive value in the breeding population.

ii) Heterozygosity has to be maintained to exploit dominance effects and keep the dominance value at a high level in the breeding population.

767 beneficial alleles in homozygous state and hence to improve the additive value, it also

768 results in a reduction of heterozygosity and the dominance value. In the worst case 769 scenario, the decrease in the dominance value over time will exceed the increase in the 770 additive value, and the rate of genetic gain will become negative due to inbreeding 771 depression. Hence, it is obvious that both the contribution of the additive value and the 772 contribution of the dominance value to genetic gain must be taken into account when 773 selecting the crossing parents of the next generation.

775 additive value and the dominance value based on the dominance degree. As the 776 dominance degree increases, the importance of the dominance value relative to the 777 additive value also increases, indicating that heterozygosity should be conserved more 
778 effectively. Optimally, a method to select new parents would automatically balance the 779 contribution of the additive and dominance components to sustain long-term genetic 780 gain without any prior knowledge about the dominance degree.

Selection of parents based on genomic predicted cross performance enabled an efficient simultaneous exploitation of additive effects and dominance an efficient simultaneous exploitation of additive effects and dominance effects by reducing the increase in inbreeding over time when the dominance degree increased. This became a crucial feature to increase genetic gain over time when the dominance degree was high.

As the dominance degree increased, selection of parents based on genomic 791 prediction of cross performance produced increasingly more genetic gain than selection 792 based on genomic estimated breeding values. By definition, the genomic estimated 793 breeding value is the sum of the average effects of the marker alleles of a genotype. 794 These average effects are predicted for all markers simultaneously by performing a 795 linear regression of the phenotypes in the training population on the marker genotypes, 796 the concept described by Falconer (1985) for a one-locus model. Although the genomic 797 estimated breeding value thereby generally captures a large part of the dominance 798 interaction (Falconer and Mackay, 1996; Hill et al., 2008), this population-based 799 predictor of the value of an individual parent for the progeny generation ignores 800 dominance deviation. 
802 fully captures both additive and dominance marker effects. It thereby enables prediction

803 of the expected genetic value of the progeny of a certain cross rather than prediction of

804 the value of an individual parent. The inclusion of non-additive effects can facilitate an

805 enhancement and an improved exploitation of non-additive genetic variation compared

806 to parent selection based on genomic estimated breeding values (Varona et al., 2018).

808 enhancement of non-additive genetic variation was a direct outcome of the reduced

809 increase in inbreeding over time. The improved exploitation of non-additive genetic

810 variation resulted from the efficiently balanced exploitation of the additive and 811 dominance value when dominance was appreciable.

813 assigned more weight to the dominance value as dominance increased without any prior

814 knowledge about the dominance degree. This was achieved by including a covariate 815 associated with genomic inbreeding (heterozygosity) in the model, which accounted for 816 directional dominance, and can be seen as an estimator for inbreeding depression 817 caused by genomic inbreeding (Xiang et al., 2016; Varona et al., 2018). As the 818 dominance degree increased, the value of crosses which maintained heterozygosity in 819 the population increased, and genomic prediction of cross performance accurately 820 predicted those crosses. 

in the additive value.

At first sight, this might seem surprising as we did not simulate overdominance.

831 Under the one-locus model with a dominance degree $<1$, the allelic combination with 832 the beneficial allele in homozygous state will result in the highest genetic value of all 833 pairwise allelic combinations. In this case, selection of parents based on the genomic 834 estimated breeding value would be an efficient strategy to increase the frequency of the 835 beneficial allele in the population over time, and hence to increase genetic gain. Only under overdominance does the heterozygote become superior to both homozygotes and 837 therefore the fixation of the allele with the higher additive value would result in a 838 reduction of the genetic value (Falconer and Mackay, 1996) 
846 an increase in the frequency of the haplotype blocks with the highest sum of average

847 effects. The heterotic effects due to pseudo-overdominance, however, are reduced, or

848 get lost, from one generation to the next. Furthermore, even haplotype blocks with

849 lower genomic estimated breeding values may contain beneficial alleles, which are 850 removed from the population through selection. As a result, genetic variance is reduced, 851 restricting long-term additive genetic gain. hand, included the heterotic potential of a cross when predicting the performance of the

854 progeny. In this way, non-additive effects due to complementation of haplotype blocks can be maintained in the population over several generations when their contribution to

856 the genetic value is high. Furthermore, by preserving haplotype blocks with lower 857 genomic estimated breeding values for a few generations, recombination will make the 858 beneficial alleles that they contain available for sustainable long-term genetic gain. Multiple crossing cycles per year using genomic prediction of cross performance without updating the prediction model can have an adverse effect on

\section{long-term genetic gain especially when the dominance degree is high}

In the two-part breeding programs with parent selection based on genomic

864 predicted cross performance, genomic inbreeding increased faster with three crossing 865 cycles per year compared to one crossing cycle per year. While using three crossing 866 cycles per year gave more genetic gain than one crossing cycle when the dominance 867 degree was low, it gave less genetic gain when the dominance degree was high. 

became crucial to ensure a sustainable, long-term exploitation of dominance effects.

871 be less efficient with three crossing cycles per year compared to one crossing cycle per 872 year: number of crosses and seedlings per cross resulted in an accelerated removal of haplotype block diversity from the breeding population. To equalize annual costs, the size of the seedling population was reduced from 12,000 to 4,000 seedlings per cross with three crossing cycles per year. Hence, the population became more susceptible to genetic drift and dominance effects due to complementation of haplotype blocks could not be maintained over multiple generations. resulted in a less efficiently balanced exploitation of additive and dominance effects. Although multiple cycles of crossing and selection per year effectively reduced the generation interval, the genomic prediction model was updated only once a year, and 886 selection of new crosses became increasingly less efficient. Assuming purely additive 887 gene action in a simulation of a line breeding program, Gaynor et al. (2017) found that 888 the increased genetic distance between the training and prediction population caused 889 selection accuracy to drop with every additional crossing cycle. Although we also 890 observed a reduction in prediction accuracy with an increased number of cycles (Fig. 891 S4), the unchanged weights assigned to additive and dominance effects by the 
892 911 effects.

prediction model contributed more strongly to the accelerated reduction of heterozygosity. While inbreeding increased with every crossing cycle, the covariate associated with genomic inbreeding in the prediction model remained unchanged for two more cycles and could not sufficiently counteract inbreeding. When the model was then updated again in the following year, the loss of heterozygosity could not be completely reversed, which became especially problematic at a high dominance degree.

maximize genetic gain in the progeny generation might not be the best method to select new parents when multiple cycles of crossing and selection per year are used. To solve this problem, we hypothesize that a strategy such as optimal contribution selection could be useful to maximize long-term genetic gain as shown by Gorjanc et al. (2017) in a two-part line breeding program with multiple crossing cycles per year.

\section{the dominance degree was high}

\section{Heterozygosity became a reliable predictor of the dominance value when}

dominance degree increased. Furthermore, prediction accuracy of the genetic value of the seedling genotypes increased as the dominance degree increased. Both prediction criteria included a non-additive term in the prediction model to capture dominance

Prediction accuracy of genomic predicted cross performance increased as the We infer that marker-based heterozygosity became an accurate predictor of nonadditive genetic effects for selection of new parents especially when the dominance degree was high. This was mostly driven by including the covariate associated with 
915 genomic inbreeding (heterozygosity) in the model, which accounted for directional

916 dominance. The two-part breeding programs especially benefited from the increase in

917 prediction accuracy when the dominance degree increased.

Not only could cross performance be predicted more accurately, but selection

919 accuracy in the seedlings also was significantly increased under high dominance

920 degrees. Both factors contributed to the two-part breeding programs with genomic

921 predicted cross performance generating the most genetic gain over time when

922 dominance was appreciable.

923

\section{Implications for other breeding programs for outbred individuals}

We expect genomic predicted cross performance could be used in other

926 breeding programs for outbred individuals, such as animal breeding programs, to

927 increase rates of genetic gain. As with clonal crops, animal breeding programs must

928 also account for the detrimental effects of inbreeding depression. Animal breeders use

929 various strategies to accomplish this ranging from rule-of-thumb recommendations to

930 avoid matings between close relatives to optimal contribution selection, a numeric

931 technique for limiting population level inbreeding (Woolliams et al., 2015). We expect

932 genomic predicted cross performance to outperform these techniques by directly

933 estimating progeny performance and thereby accounting for inbreeding depression in a

934 purely data-driven manner, given the prediction model is constantly updated. However,

935 when multiple cycles of crossing and selection per year are used without updating the

936 prediction model, genomic prediction of cross performance to maximize genetic gain

937 in the progeny generation might not be the best method to select new parents. In this 
938 case, implementing a strategy like optimal contribution selection might be useful to

939 maximize long-term genetic gain, outlining an important topic for further research.

940

941

942

Acknowledgments

943

The authors acknowledge the financial support from Innovate UK (132748).

944

945

Conflict of interest

946

The authors declare that they have no conflict of interest.

947

948

949

950

951

952

953

954 


\section{Crossing}

\section{Seedling stage unreplicated Seedlings}

Clonal stage 1 unreplicated clones; training population for genomic selection

Clonal stage 2-5 replicated clones

\section{Variety release}
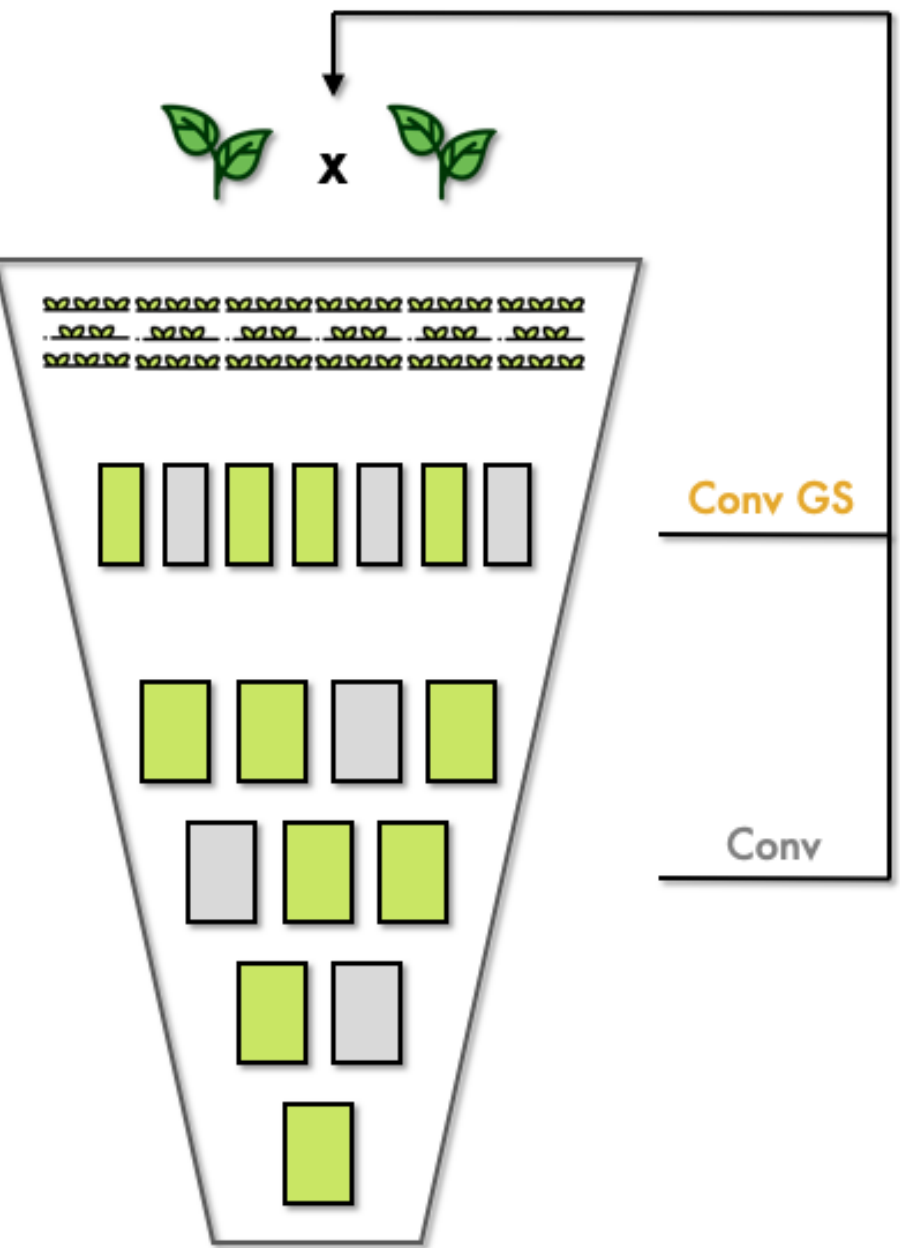

956

Figure 1 Schematic overview of the conventional breeding program and

958 the conventional breeding program with genomic selection. The conventional

959 breeding program (Conv) was used in the burn-in breeding phase and served as a

960 control in the future breeding phase. In the conventional breeding program, parents

961 were selected in clonal stages $2-5$. The conventional breeding program with genomic

962 selection reduced the generation interval to two years by selecting parents in clonal

963 stage 1 based on either genomic estimated breeding values or genomic predicted cross

964 performance. The genotypes in clonal stage 1 served as training population. 


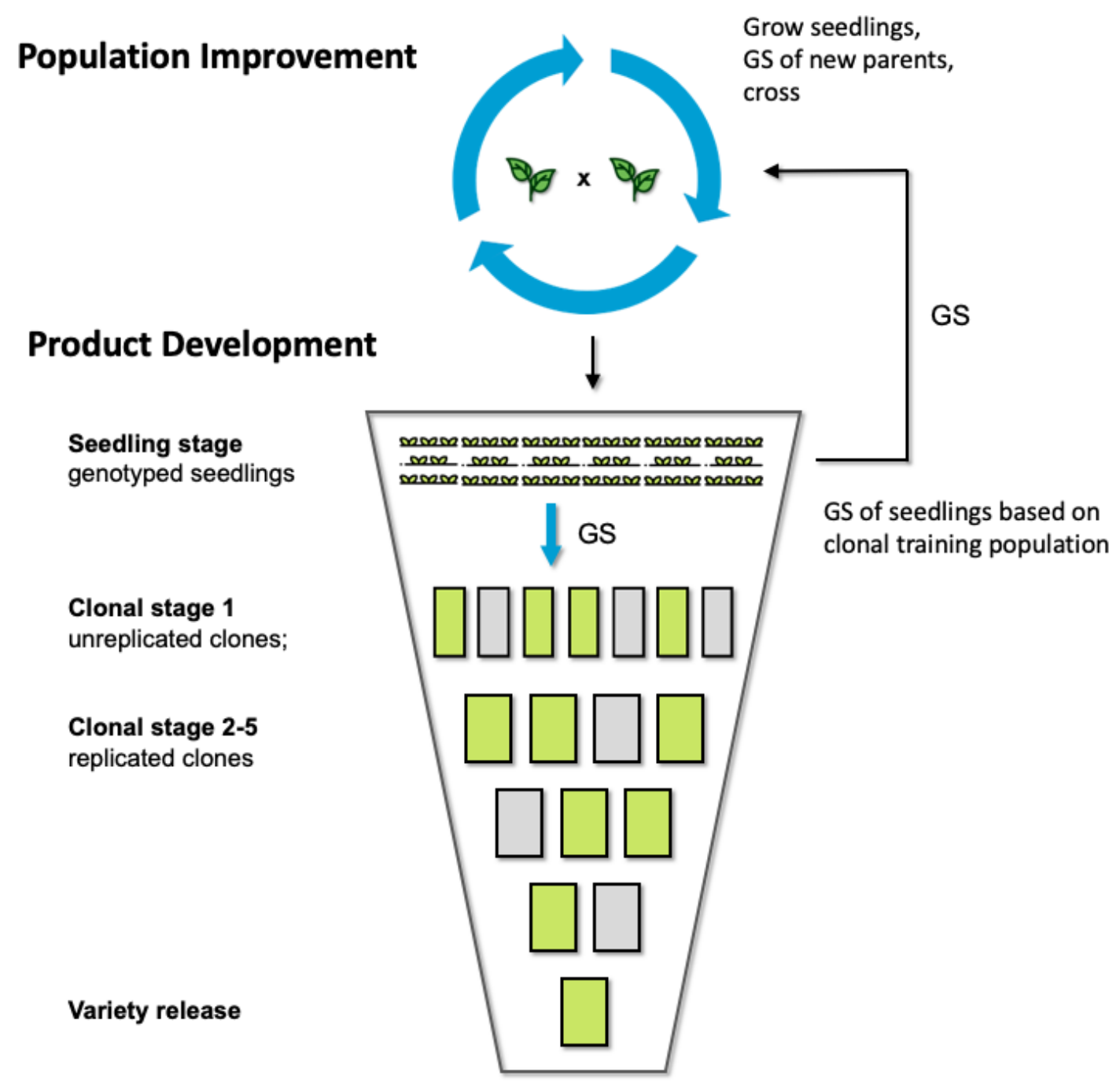

Figure 2 Schematic overview of the two-part breeding program. The two-

968 part breeding program reorganized the conventional breeding program into i) a

969 population improvement component to develop improved germplasm through rapid

970 recurrent genomic selection; and ii) a product development component to identify the

971 best performing genotypes. The population improvement component allows to have

972 multiple cycles of crossing and selection per year before the seedlings are advanced to

973 the product development component based on their genomic estimated genetic values.

974 New parents during population improvement were selected based on either genomic

975 estimated breeding values or genomic predicted cross performance. 


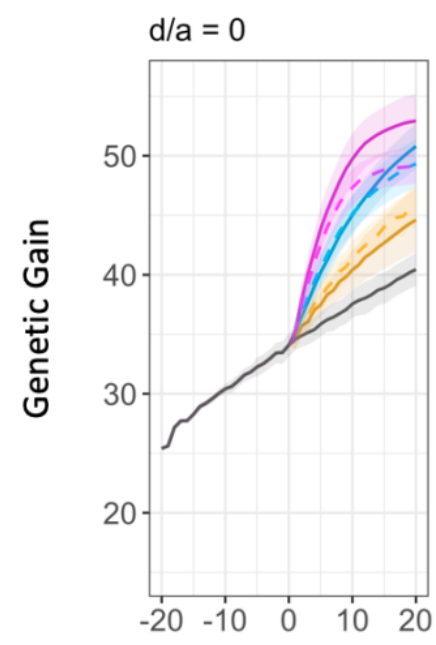

976
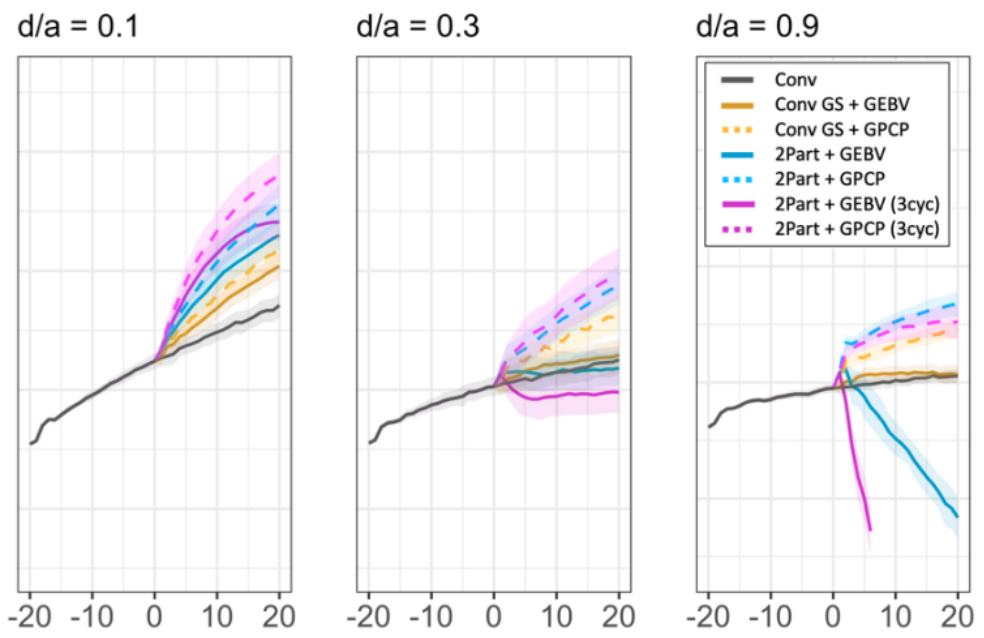

Year

Figure 3 Genetic gain of the simulated breeding programs under different dominance degrees $(\mathbf{d} / \mathbf{a})$. In each panel, genetic gain is plotted as mean genetic value in clonal stage 1 for the entire burn-in breeding phase and the future breeding phase. Each line shows the mean genetic value for the 10 simulated replications and the shading shows the $95 \%$ confidence intervals. The different types of breeding program are shown in different colours. The conventional breeding program (Conv) is gray. The conventional breeding program with genomic selection (Conv GS) is yellow. The twopart breeding program with genomic selection (2Part) is shown in blue with one crossing cycle per year and in purple with three crossing cycles per year. The two types of parent selection were shown in different line-styles. Selection based on Genomic Estimated Breeding Value (GEBV) is shown by continuous lines. Selection based on Genomic Prediction of Cross Performance (GPCP) is shown by dashed lines. 


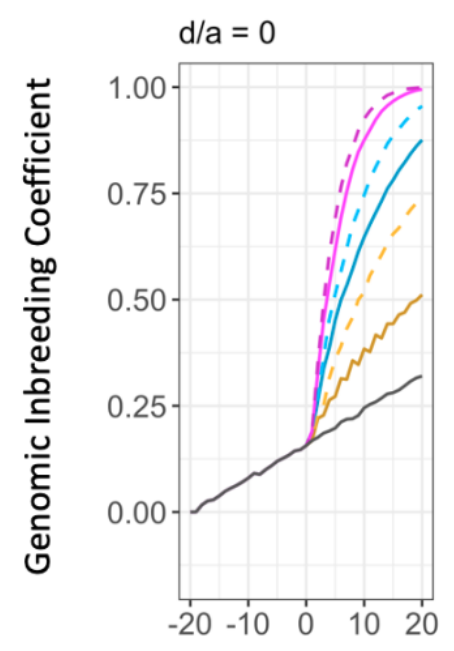

992

993

994

995

996

997

998

999

1000

1001

1002

1003

1004

1005

1006

.
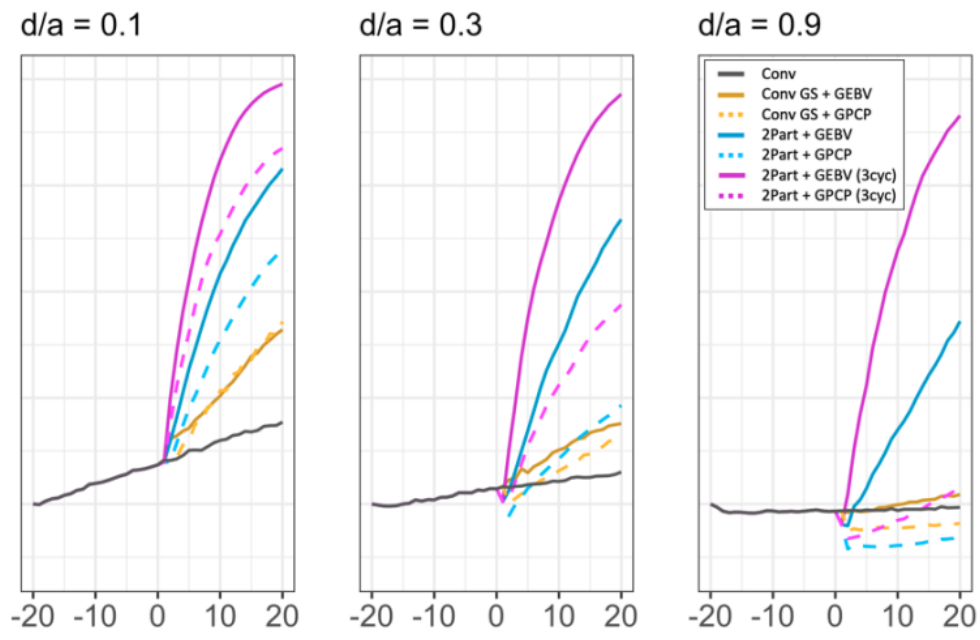

Year

\section{Figure 4 Genomic inbreeding coefficient of the simulated breeding} programs under different dominance degrees (d/a). In each panel, the genomic inbreeding coefficient is plotted in clonal stage 1 for the entire burn-in breeding phase and the future breeding phase. Each line shows the mean genomic inbreeding coefficient for the 10 simulated replications. The different types of breeding program are shown in different colours. The conventional breeding program (Conv) is gray. The conventional breeding program with genomic selection (Conv GS) is yellow. The twopart breeding program with genomic selection (2Part) is shown in blue with one crossing cycle per year and in purple with three crossing cycles per year. The two types of parent selection were shown in different line-styles. Selection based on Genomic Estimated Breeding Value (GEBV) is shown by continuous lines. Selection based on Genomic Prediction of Cross Performance (GPCP) is shown by dashed lines. 


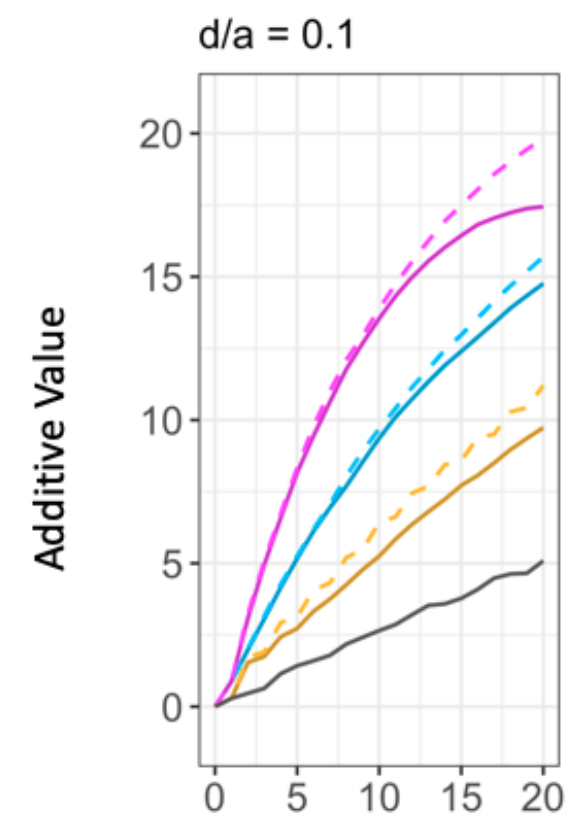

$$
\mathrm{d} / \mathrm{a}=0.3
$$

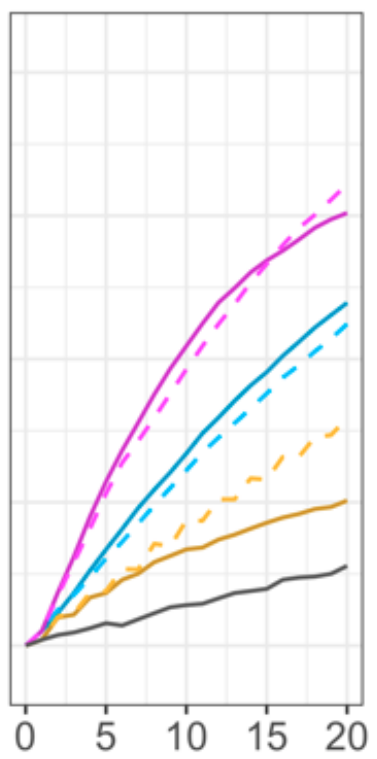

$\mathrm{d} / \mathrm{a}=0.9$
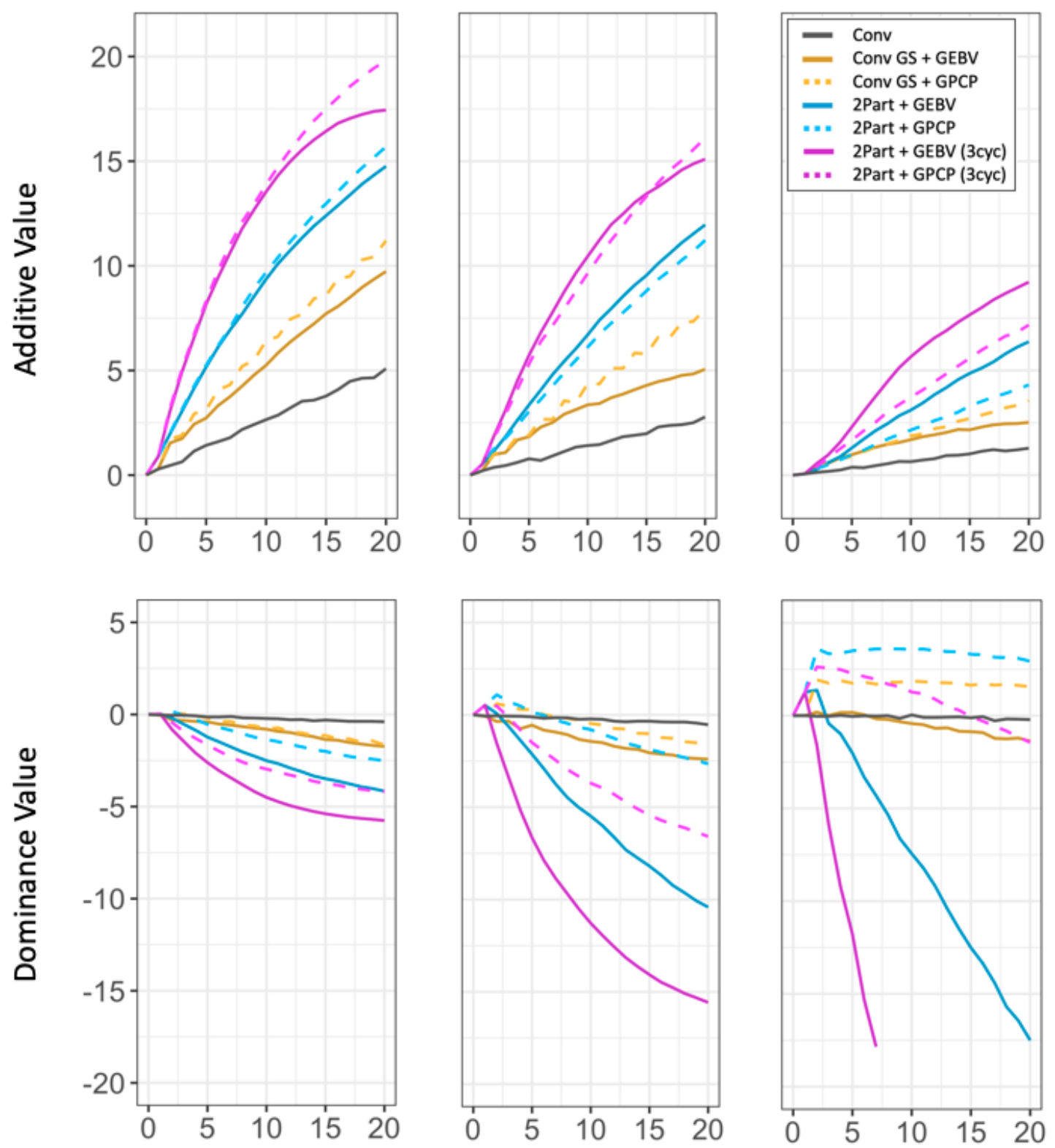

1008

Figure 5 Additive values and the dominance values of the simulated

1010 breeding programs under different dominance degrees (d/a). In each of the upper

1011 panels (a-c), the additive values are plotted in clonal stage 1 for the future breeding

1012 phase. The lower panels (d-f) plot the dominance values. Each line shows the mean

1013 value for the 10 simulated replications. The different types of breeding program are

1014 shown in different colours. The conventional breeding program (Conv) is gray. The

1015 conventional breeding program with genomic selection (Conv GS) is yellow. The two-

1016 part breeding program with genomic selection (2Part) is shown in blue with one 
1017

1018

1019

1020

1021

1022

1023

crossing cycle per year and in purple with three crossing cycles per year. The two types of parent selection were shown in different line-styles. Selection based on Genomic Estimated Breeding Value (GEBV) is shown by continuous lines. Selection based on Genomic Prediction of Cross Performance (GPCP) is shown by dashed lines. Additive values and dominance values at the beginning of the future breeding phase (year 0) were centred at zero.
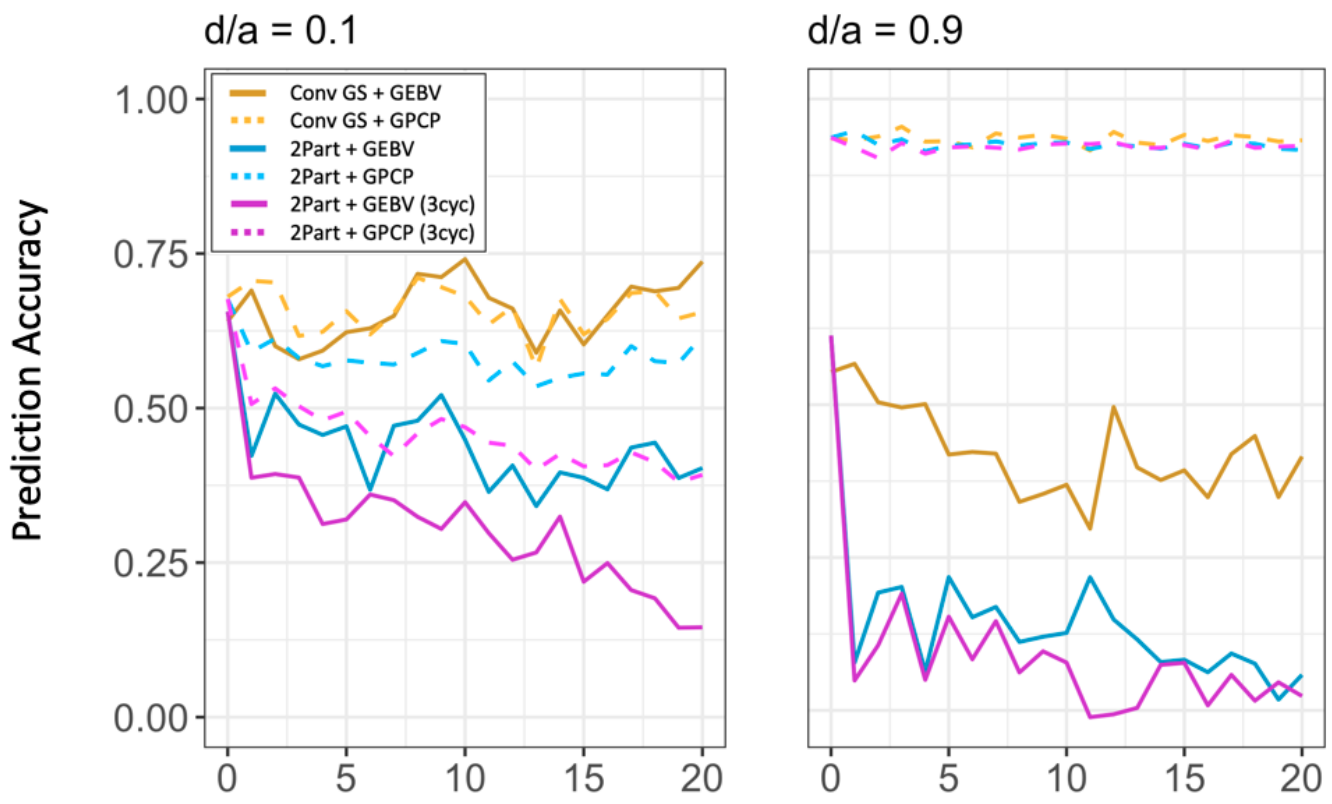

Year

Figure 6 Prediction accuracy for selection of new parents under different dominance degrees (d/a). In each panel, prediction accuracy is plotted for the future breeding phase of the breeding programs with genomic selection. Each line shows the mean prediction accuracy for the 10 simulated replications. The different types of breeding program are shown in different colours. The conventional breeding program with genomic selection (Conv GS) is yellow. The two-part breeding program with genomic selection (2Part) is shown in blue with one crossing cycle per year and in purple with three crossing cycles per year. The two types of parent selection were shown in different line-styles. Selection based on Genomic Estimated Breeding Value (GEBV) is shown by continuous lines. Selection based on Genomic Prediction of Cross Performance (GPCP) is shown by dashed lines. Prediction accuracy was measured in 
1036

1037

1038

1039

the seedling stage for the two-part breeding programs and in clonal stage 1 for the conventional breeding program with genomic selection.

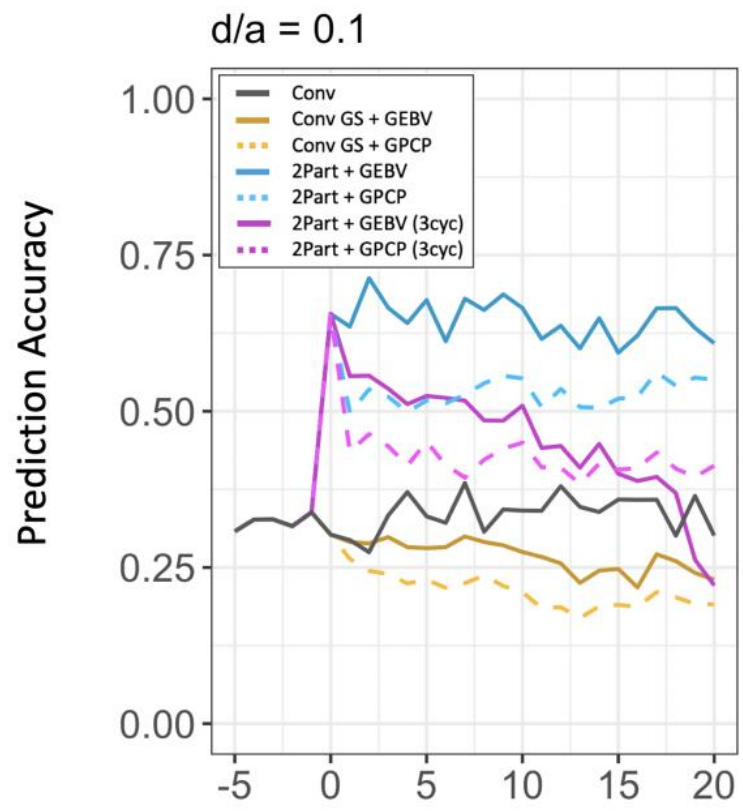

$\mathrm{d} / \mathrm{a}=0.9$

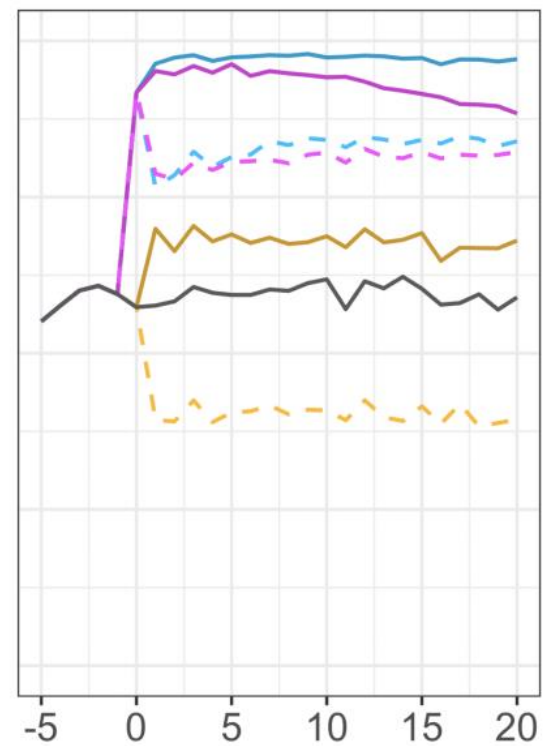

Year

Figure 7 Prediction accuracy for the total genetic value of the seedlings under different dominance degrees (d/a). In each panel, prediction accuracy is plotted in the seedling stage for the entire burn-in breeding phase and the future breeding phase. Each line shows the mean prediction accuracy for the 10 simulated replications. The different types of breeding program are shown in different colours. The conventional breeding program (Conv) is gray. The conventional breeding program with genomic selection (Conv GS) is yellow. The two-part breeding program with genomic selection (2Part) is shown in blue with one crossing cycle per year and in purple with three crossing cycles per year. The two types of parent selection were shown in different line-styles. Selection based on Genomic Estimated Breeding Value (GEBV) is shown by continuous lines. Selection based on Genomic Prediction of Cross Performance (GPCP) is shown by dashed lines. 


\section{References}

1054 Bassil, N.V., T.M. Davis, H. Zhang, S. Ficklin, M. Mittmann, et al. 2015. Development

1055

1056

1057

1058

1059

1060

1061

1062

1063

1064

1065

1066

1067

1068

1069

1070

1071

1072

1073

1074

and preliminary evaluation of a $90 \mathrm{~K}$ Axiom ${ }^{\circledR}$ SNP array for the allo-octoploid cultivated strawberry Fragaria $\times$ ananassa. BMC Genomics 16(1). doi: 10.1186/s12864-015-1310-1.

Bingham, E.T. 1998. Role of Chromosome Blocks in Heterosis and Estimates of Dominance and Overdominance. In: Larnkey, K.R. and Staub, J.E., editors, CSSA Special Publications. Crop Science Society of America, Madison, WI, USA. p. $71-87$

Bingham, E.T., R.W. Groose, D.R. Woodfield, and K.K. Kidwell. 1994. Complementary Gene Interactions in Alfalfa are Greater in Autotetraploids than Diploids. Crop

Sci. 34(4): 823-829. doi: 10.2135/cropsci1994.0011183X003400040001x.

Bisognin, D.A. 2011. Breeding vegetatively propagated horticultural crops. Crop Breed. Appl. Biotechnol. 11(spe): 35-43. doi: 10.1590/S198470332011000500006 .

Bradshaw, J. 2016. Plant breeding: past, present and future. Springer, Cham.

Chen, G.K., P. Marjoram, and J.D. Wall. 2009. Fast and flexible simulation of DNA sequence data. Genome Res. 19(1): 136-142. doi: 10.1101/gr.083634.108.

Crossa, J., P. Pérez-Rodríguez, J. Cuevas, O. Montesinos-López, D. Jarquín, et al. 2017. Genomic Selection in Plant Breeding: Methods, Models, and Perspectives. Trends Plant Sci. 22(11): 961-975. doi: 10.1016/j.tplants.2017.08.011. 
1075

1076

1077

1078

1079

1080

1081

1082

1083

1084

1085

1086

1087

1088

1089

1090

1091

1092

1093

1094

1095

1096

van Dijk, T., G. Pagliarani, A. Pikunova, Y. Noordijk, H. Yilmaz-Temel, et al. 2014. Genomic rearrangements and signatures of breeding in the allo-octoploid strawberry as revealed through an allele dose based SSR linkage map. BMC Plant Biol. 14(1): 55. doi: 10.1186/1471-2229-14-55.

Falconer, D.S. 1985. A note on Fisher's 'average effect' and 'average excess.' Genet. Res. 46(3): 337-347. doi: 10.1017/S0016672300022825.

Falconer, D.S., and T.F.C. Mackay. 1996. Introduction to quantitative genetics. 4. ed., [16. print.]. Pearson, Prentice Hall, Harlow.

Gaynor, R.C., G. Gorjanc, A.R. Bentley, E.S. Ober, P. Howell, et al. 2017. A Two-Part Strategy for Using Genomic Selection to Develop Inbred Lines. Crop Sci. 57(5): 2372-2386. doi: 10.2135/cropsci2016.09.0742.

Gaynor, R.C., G. Gorjanc, D. Wilson, and J.M. Hickey. 2019. AlphaSimR: Breeding Program Simulations.

Gemenet, D.C., and A. Khan. 2017. Opportunities and Challenges to Implementing Genomic Selection in Clonally Propagated Crops. In: Varshney, R.K., Roorkiwal, M., and Sorrells, M.E., editors, Genomic Selection for Crop Improvement. Springer International Publishing, Cham. p. 185-198

Goddard, M. 2009. Genomic selection: prediction of accuracy and maximisation of long term response. Genetica 136(2): 245-257. doi: 10.1007/s10709-008-93080 .

Goddard, M.E., and B.J. Hayes. 2007. Genomic selection: Genomic selection. J. Anim. Breed. Genet. 124(6): 323-330. doi: 10.1111/j.1439-0388.2007.00702.x. 
1097 Gorjanc, G., R.C. Gaynor, and J.M. Hickey. 2017. Optimal cross selection for longterm genetic gain in two-part programs with rapid recurrent genomic selection. bioRxiv: 227215. doi: 10.1101/227215.

Grüneberg, W., R. Mwanga, M. Andrade, and J. Espinoza. 2009. Selection methods.

Hill, W.G., M.E. Goddard, and P.M. Visscher. 2008. Data and Theory Point to Mainly 322. Additive Genetic Variance for Complex Traits. PLOS Genet. 4(2): e1000008. in animal breeding. Anim. Front. 6(1): 6-14. doi: 10.2527/af.2016-0002. al. 2009. A genetic linkage map of the cultivated strawberry Fragaria $\times$ ananassa

1112 Sargent, D.J., Y. Yang, N. Šurbanovski, L. Bianco, M. Buti, et al. 2016. HaploSNP affinities and linkage map positions illuminate subgenome composition in the octoploid, cultivated strawberry (Fragaria×ananassa). Plant Sci. 242: 140-150. doi: 10.1016/j.plantsci.2015.07.004. 
bioRxiv preprint doi: https://doi.org/10.1101/2020.06.15.152017; this version posted June 15, 2020. The copyright holder for this preprint (which was not certified by peer review) is the author/funder, who has granted bioRxiv a license to display the preprint in perpetuity. It is made available under aCC-BY-NC-ND 4.0 International license.

1118

1119

1120

1121

1122

1124

1125 Xiang, T., O.F. Christensen, Z.G. Vitezica, and A. Legarra. 2016. Genomic evaluation Using Genome-Wide Dense Single Nucleotide Polymorphism Markers. PLOS ONE 7(9): e45293. doi: 10.1371/journal.pone.0045293.

Varona, L., A. Legarra, M.A. Toro, and Z.G. Vitezica. 2018. Non-additive Effects in Genomic Selection. Front. Genet. 9. doi: 10.3389/fgene.2018.00078.

Woolliams, J.A., P. Berg, B.S. Dagnachew, and T.H.E. Meuwissen. 2015. Genetic contributions and their optimization. J. Anim. Breed. Genet. 132(2): 89-99. doi: $10.1111 / j b g .12148$.

1126 by including dominance effects and inbreeding depression for purebred and crossbred performance with an application in pigs. Genet. Sel. Evol. 48(1). doi:

1128 10.1186/s12711-016-0271-4. 